\title{
Cancer malignancy is correlated with up-regulation of PCYT2-mediated glycerol phosphate
} modification of $\alpha$-dystroglycan

Fumiko Umezawa ${ }^{1,2}$, Makoto Natsume ${ }^{3}$, Shigeki Fukusada ${ }^{3}$, Kazuki Nakajima ${ }^{4}$, Fumiya Yamasaki ${ }^{5}$,

Hiroto Kawashima ${ }^{5}$, Chu-Wei Kuo ${ }^{6}$, Kay-Hooi Khoo ${ }^{6}$, Takaya Shimura ${ }^{3}$, Hirokazu Yagi2,", Koichi

$$
\text { Kato }^{1,2, *}
$$

${ }^{1}$ Exploratory Research Center on Life and Living Systems (ExCELLS) and Institute for Molecular Science (IMS), National Institutes of Natural Sciences, Okazaki, Japan

${ }^{2}$ Department of Structural Biology and Biomolecular Engineering, Graduate School of Pharmaceutical Sciences and ${ }^{3}$ Department of Gastroenterology and Metabolism, Graduate School of Medical

Sciences, Nagoya City University, Nagoya, Japan

${ }^{4}$ Center for Joint Research Facilities Support, Research Promotion and Support Headquarters, Fujita Health University, Toyoake, Aichi 470-1192, Japan

${ }^{5}$ Laboratory of Microbiology and Immunology, Graduate School of Pharmaceutical Sciences, Chiba University, Chiba, Japan

${ }^{6}$ Institute of Biological Chemistry, Academia Sinica, Taipei, Taiwan

*Addresses for correspondence: Hirokazu Yagi, Ph.D., Department of Structural Biology and Biomolecular Engineering, Graduate School of Pharmaceutical Sciences, Nagoya City University, 3-1 Tanabe-dori, Mizuho-ku, Nagoya 467-8603, Japan, Tel: +81-52-836-3448, Fax: +81-52-836-3450, email: hyagi@phar.nagoya-cu.ac.jp and Koichi Kato, PhD., Exploratory Research Center on Life and Living Systems and Institute for Molecular Science, National Institutes of Natural Sciences, 5-1 Higashiyama, Myodaiji, Okazaki, Aichi 444-8787, Japan, Tel: +81-564-59-5225, Fax: +81-564-595224, e-mail:kkatonmr@ims.ac.jp

\section{Abstract}

The dystrophin-glycoprotein complex connects the cytoskeleton with base membrane components such as laminin through unique O-glycans displayed on $\alpha$-dystroglycan ( $\alpha$-DG). Genetic impairment of elongation of these glycans causes congenital muscular dystrophies. We previously identified that glycerol phosphate (GroP) can cap the core part of the $\alpha$-DG Oglycans and terminate their further elongation. This study examined the possible roles of the GroP modification in cancer malignancy, focusing on colorectal cancer. We found that the GroP modification critically depends on PCYT2, which serves as CDP-Gro synthase.

Furthermore, we identified a significant positive correlation between cancer progression and GroP modification, which also correlated positively with PCYT2 expression. Moreover, we demonstrate that GroP modification promotes the migration of cancer cells. Based on these 
findings, we propose that the GroP modification by PCYT2 disrupts the glycan-mediated cell adhesion to the extracellular matrix and thereby enhances cancer metastasis. Thus, the present study suggests the possibility of novel approaches for cancer treatment by targeting the PCYT2-mediated GroP modification.

Key words: Cancer malignancy; CDP-glycerol, $\alpha$-Dystroglycan, Glycerol phosphate modification; Laminin-binding glycans; PCYT2 


\section{Introduction}

Epithelial cells interact with the basement membrane, a sheet-like type of extracellular matrix (ECM), through cell adhesion molecules, which regulate cellular growth, motility, and differentiation by integrating signals from ECM or soluble factors $(1,2)$. One of the most extensively studied cell adhesion molecules is $\alpha$-dystroglycan $(\alpha-D G)$, which is displayed on epithelial cell surfaces as a component of the dystrophin-glycoprotein complex $(3,4)$. This complex connects the cytoskeleton with basement membrane components such as laminin, agrin, and perlecan and plays an essential role in epithelium development and tissue organization. The connection critically depends on unique O-glycans, termed laminin-binding glycans, attached to the N-terminal region of the mucin-like domain of $\alpha$-DG (5-7).

The outer regions of laminin-binding glycans consist of $\alpha 1-3 \mathrm{Xyl} \beta 1-3 \mathrm{GlcA}$ repeat sequences formed by a dual functional glycosyltransferase LARGE and responsible for the interactions with the base membrane components (8) The inner part of laminin-binding glycan is constituted from the reducing terminal 6-phosphorylated core M3 structure, i.e., GalNAc $\beta 1$ 3GlcNAc 31 -4Man-6-phosphate, and a tandem ribitol-5-phosphate (Rbo5P) structure followed by a $\beta 1-4 X y 1 \beta 1-4 G l c A$ disaccharide. Besides LARGE, at least nine different glycosyltransferases are involved in forming the laminin-binding glycans (9-16) Mutational dysfunction of either of these enzymes impairs the formation of laminin-binding glycans, disrupting the $\alpha$-DG-ECM interactions, causing congenital muscular dystrophies (CMD) designated as $\alpha$-dystroglycanopathies. Among these enzymes, Fukutin (FKTN) and Fukutinrelated protein (FKRP) catalyze the first and second steps of Rbo5P transfer, respectively. This donor substrate is synthesized by CDP-L-ribitol pyrophosphorylase A (CRPPA/ISPD) $(15,17-19)$.

We recently found that glycerol phosphate (GroP) modifies the 6-phosphorylated core M3 structure without further elongation (20). The dual action of FKTN catalyzes this novel modification and, therefore, competes for the first Rbo5P conjugation $(20,21)$. Furthermore, the GroP modification is enhanced by elevation of intracellular CDP-Gro level, which can be artificially realized by overexpression of TagD, a CDP-Gro synthase from Gram-positive bacteria $(22,23)$, resulting in a concomitant decrease of the laminin-binding glycans $(24)$. These findings raise the possibility that GroP serves as a terminator of the laminin-binding glycan elongation. However, the physiological and pathological functions of this modification remain largely unknown.

This study examined the possible roles of the GroP modification in cancer malignancy because defects in the laminin-binding glycans of $\alpha$-DG have been found not only in CMD but also in various carcinomas $(25,26)$. We revealed that the GroP modification was promoted in several cancer tissues and addressed the molecular mechanism and pathological significance of this modification. 


\section{Results}

\section{GroP modification is enhanced in cancer tissues}

We previously established a monoclonal antibody DG2, reactive with GroP-modified $\alpha$ DG (24). Using this antibody, we probed the GroP modification in various normal and cancerous tissues. Several cancerous tissues, including the bladder, uterus, ovary, and colon, tested positive for immunostaining compared to their normal counterparts (Supplementary Table 1). Focusing on colorectal cancer, we investigated a relationship between degrees of GroP modification and malignancy (Figure 1). GroP expression in human CRC tissues was analyzed using immunohistochemistry with DG2 for primary tumor tissues with surgical resection. Representative images of DG2 staining are shown in Figure 1A. The 100 CRC patients consisted of 14 patients in stage 0, 20 patients in stage I, 21 patients in stage II, 22 patients in stage III and 23 patients in stage IV. The stage IV CRC showed a significantly higher rate of positive GroP expression than the other stages (stage $0,14 \%(2 / 12)$; stage I, $25 \%(5 / 20)$; stage II, $43 \%$ (9/21); stage III, $36 \%(8 / 22)$; stage IV, $74 \%(17 / 23)$ ) (Figure 1B). Notably, no immunoreactivity to DG2 was observed in normal colorectal tissues. These data indicated that GroP modification is promoted as cancer progresses.

\section{GroP modification promotes cancer migration}

We examined the possible effects of the GroP modification enhanced by TagD overexpression on the proliferation and migration of HCT116 cells as a model of colorectal cancer cells. While the WST-8 assay showed virtually no effect on cell proliferation, the migration ability was enhanced upon TagD overexpression, as demonstrated by woundhealing and transwell assays (Figure 2). These data indicated that enhancement of the GroP modification promoted cancer cell migration, which could be caused by an increase in CDPGro amount in cells.

\section{PCYT2 synthesizes CDP-Gro}

We next attempted to identify mammalian enzymes that catalyze the CDP-Gro synthesis assuming their homology with bacterial TagD. From the human genome database, we found five genes, CDS1, CDS2, PCYT1A, PCYT1B, and PCYT2, that share $>30 \%$ homology with the TagD gene (Table 1). We produced recombinant proteins encoded by these genes in HCT116 cells and evaluated their CDP-Gro synthetic activity in vitro (Figure 3A). Consequently, we found that only PCYT2 could synthesize CDP-Gro, while the remaining four proteins had no activity. To date, PCYT2 have been characterized as ethanolaminephosphate cytidylyltransferase catalyzing the formation of CDP-ethanolamine (CDP-Etn)(27, 28). This enzyme has two major splicing isoforms, PCYT2 $\alpha$ and PCYT2 $\beta$, which were shown to be comparable in terms of the enzymatic activities as CDP-Gro synthase as well as CDP-Etn synthase (Figure 3B and Table 2) 
Furthermore, to investigate whether PCYT2 actually synthesizes CDP-Gro in cells, we established PCYT2-KO cells (Supplementary Figure 1) and quantified intracellular CDP-Gro and CDP-Etn in the cells based on their extraction ion chromatograms (Figure 4A). As expected, CDP-Etn was markedly reduced in the PCYT2-KO cells (Figure 4B). Moreover, CDP-Gro was undetectable in the PCYT2-KO cells. Furthermore, the CDP-Gro level was recovered in the PCYT2-KO cells rescued by overexpressing PCYT2 (Figure 4B). These results indicate that PCYT2 is the enzyme responsible for the synthesis of CDP-Gro in mammalian cells.

\section{GroP modification of $\alpha$-DG critically depends on PCYT2}

We overexpressed the Fc-fused $\alpha$-DG recombinant protein with amino acid substitution and deletion of $\alpha$-DG [ $\alpha$-DG373(T322R)-Fc] in the wild-type (WT) and PCYT2-KO cells. We analyzed their GroP modifications by nanospray liquid chromatography-tandem mass spectrometry (LC-MS/MS) (Figure 5). The data demonstrated that the GroP modification of laminin-binding glycans was not detected in the PCYT2-KO cells in contrast to the WT cells. In addition, the overexpression of PCYT2 resulted in a significant reduction in the lamininbinding glycans on $\alpha$-DG (Figure 6). These data suggested the critical involvement of PCYT2 in the GroP modification of $\alpha$-DG by supplying CDP-Gro as a donor substrate.

We detected the expression of PCYT2 in serial sections of stage IV CRC tissues using the anti-PCYT2 antibody. Of 23 stage III CRCs, 13 CRCs revealed positive expression of PCYT2. Interestingly, the immunostaining score was significantly correlated between GroP and PCYT2 expressions in the CRC tissues $(\mathrm{r}=0.614, P=0.002)$ (Figure 7).

\section{Discussion}

The glycan-mediated cell-cell communications provide the basis of the higher functions of our body, which are represented by the immune and nervous systems $(29,30)$. Therefore, the glycans have to be properly displayed on cell surfaces, and glycosylation abnormalities cause disorders of cellular society, resulting in severe pathological symptoms as exemplified cancer malignant transformation (31). The glycan formation involves a series of glycosyltransferases whose up- and down-regulations can control glycan elongation and consequent cellular interactions and, moreover, sophisticate functions (32). In the case of laminin-binding Oglycan formation on $\alpha-D G$, at least nine different glycosyltransferases play indispensable roles and their misfunctions result in CMD. Recently, we found that GroP modification provides an alternative mode of regulation of elongation of the $\alpha$-DG O-glycans by capping the core M3 structure (20). This modification is catalyzed by the Janus-like enzyme FKTN, which also transfers Rbo5P and thereby positively contributes to the glycan elongation (21), suggesting some additional mechanism for selectively regulating the modification by GroP as a glycan termination factor. 
This study identified PCYT2 as a CDP-Gro synthase and that the GroP modification critically depends on this enzyme. We furthermore found a significantly positive correlation between colorectal cancer progression and GroP modification, which also correlated positively with PCYT2 expression. Moreover, we demonstrate that GroP modification promotes the migration of cancer cells. Finally, we integrate these findings into a cancer metastasis model wherein the GroP modification by PCYT2 abrogates the glycan-mediated cell adhesion to ECM, thereby promoting migration and invasion of cancer cells.

In addition, it has been pointed out that inadequate expression of the laminin-binding glycans may activate the integrin-mediated AKT/ERK pathway involved in cancer migration (33). Integrins are known to be competitive with $\alpha$-DG in terms of laminin binding. Therefore, the loss of the laminin-binding glycans of $\alpha$-DG results in the promotion of the integrin-laminin interactions thereby enhancing the signaling leading to cancer metastasis.

Thus, our findings raise the possibility of novel approaches for cancer treatment by antagonizing GroP modification. Particularly, PCYT2 can be a potential target for developing anti-cancer drugs. However, the present study indicates that PCYT2 is also a dual-function enzyme that synthesizes both CDP-Gro and CDP-Etn, known to be a vital precursor in the biosynthesis of phosphatidylethanolamine (34). Therefore, it is important to explore how these two enzymatic activities are regulated from an anti-cancer drug discovery perspective. An alternative approach will be developing therapeutic antibodies targeting the GroP groups displayed on malignant cancer cells.

The present study highlights the pathological aspect of GroP modification in the context of cancer metastasis. Conversely, it is also important to elucidate the physiological roles of this modification. Lymphocytes need to be freed from the adhesion to the ECM for migrating throughout our body. Indeed, T cells have been reported to lose their laminin-binding glycans upon maturation (35). An intriguing possibility is that the PCYT2-mediated GroP modification is involved in such regulations. Further elucidation of the regulatory mechanisms of this modification system will contribute to a deeper understanding of the physiological control of cell adhesion and also to develop therapeutic approaches for a disorder of the system.

While preparing this manuscript, Dr. Endo and his coworkers published a report showing that PCYT2 was a mammalian CDP-Gro synthase (36), consistent with our findings.

\section{Experimental procedures}

\section{Antibodies}

The following antibodies were used in this study: anti-Flag (M2) mouse IgG monoclonal antibody (mAb) (Sigma-Aldrich, St. Louis, MO); anti-c-Myc (9E10) mouse IgG mAb 
(Wako, Osaka, Japan); anti- $\alpha$-Dystroglycan (IIH6C4) mouse IgM mAb (Millipore, Billerica, MA); anti-PCYT2 (14827) rabbit IgG polyclonal antibody (pAb) (Proteintech, Rosemont,

IL); anti- $\beta$-actin mouse IgG mAb (Sigma-Aldrich); HRP-conjugated anti-mouse IgM mAb (Thermo Fisher Scientific, Waltham, MA); HRP-conjugated anti-mouse IgG mAb (Invitrogen, Carlsbad, CA); HRP-conjugated anti-mouse IgG antibody (Dako, Glostrup, Denmark); HRP-conjugated anti-rabbit IgG polyclonal antibody (Cell Signaling, Danvers, MA); mouse monoclonal IgM Ab reactive with GroP-modified $\alpha$-DG, DG2 (24).

\section{Patients}

One hundred colorectal cancer (CRC) patients who underwent surgical and endoscopic resection at Nagoya City University Hospital from March 2018 to December 2019 were enrolled in the present study. The clinical stage was determined based on the final pathological diagnosis after resection, according to the 7th edition of the UICC-TNM classification. The study protocol was approved by the institutional review board (IRB) of Nagoya City University (IRB \#. 60-19-0047), and it was conducted following the ethical guidelines of the 1975 Declaration of Helsinki (6 $6^{\text {th }}$ revision, 2008).

\section{Immunohistochemistry}

Immunohistochemical staining was performed using serial sections of each sample as follows. All tumor samples were resected from primary CRC tissues, fixed in formalin, and embedded in paraffin. Consecutive sections (4- $\mu \mathrm{m}$ thick) were deparaffinized and dehydrated. After inhibition of endogenous peroxidase activity by immersion in 3\% $\mathrm{H}_{2} \mathrm{O}_{2} /$ methanol solution, antigen retrieval was achieved by heating the samples in $10 \mathrm{mM}$ citrate buffer $\left(\mathrm{pH} \mathrm{6.0)}\right.$ in a microwave oven for $10 \mathrm{~min}$ in at $98^{\circ} \mathrm{C}$. Sections were then incubated with primary antibodies against $20 \mu \mathrm{g} / \mathrm{ml}$ of the anti-GroP DG2 antibody and antiPCYT2 antibody at dilution of 1:200. After thorough washing, samples were incubated with biotinylated secondary antibodies and then with avidin-biotin horseradish peroxidase complexes. Finally, immune complexes were visualized by incubation in 3,3'diaminobenzidine tetrachloride. Nuclear counterstaining was accomplished using Mayer's hematoxylin. All immunostained specimens were assessed by an independent observer blinded to all clinical information. When more than $10 \%$ of all cancer cells in each section were stained, the staining intensity score was classified as negative (0), weak (1), moderate (2), or strong (3). Finally, immunostaining scores of (2) and (3) were defined as positive. In 
the statistical analysis, the binary data were analyzed using the chi-squared test or Fisher's exact probability test, as appropriate. The nonparametric Spearman's rank correlation coefficient $(r)$ was used as a correlation measure. All statistics were calculated using SPSS Statistics version 25 (IBM Corp., Tokyo, Japan). A two-tailed $P$ value of less than 0.05 was considered statistically significant.

\section{cDNA construction}

The expression plasmid for the $\alpha$-DG373(T322R)-Fc was constructed as previously described (20). In this plasmid vector, the coding sequence of human $\alpha-D G$ (1-373) with amino acid substitution of threonine with arginine at position 322 was subcloned into pEF-Fc. For Flagtagged expression vector of TagD was constructed as previously described (24). In the expression plasmid for Flag-tagged PCYT2 (Uniprot KB: Q99447-1) recombinant protein, the coding sequence of human PCYT2 with flag peptide (DYKDDDDK) purchased from addgene (\#81074) was cloned into the pCAG-Neo vector (WAKO). The expression plasmids for Myc-tagged CDS1 (UniProt KB: P98191), CDS2 (UniProt KB: Q99L43), PCYT1A (UniProt KB: P49586), and PCYT1B (UniProt KB: Q811Q9) recombinant proteins were purchased from Origene. In bacterial expression plasmids for His6-tagged PCYT2 $\beta$ (Uniprot KB: Q99447-1) and PCYT2 $\alpha$ (Uniprot KB: Q99447-3) recombinant proteins, the coding sequences were cloned into pColdI vector (Takara Bio Inc.).

\section{Cell culture and transfection}

HCT116 (ATCC) cells were maintained in Dulbecco's modified Eagle's medium (DMEM)high glucose (Gibco) supplemented with 10\% fetal bovine serum (FBS) (Cell Culture Technologies), for $37^{\circ} \mathrm{C}$, at $5 \% \mathrm{CO}_{2}$. For cDNA transfection, cells were grown overnight and transfected using polyethyleneimine "Max" (Polysciences, Inc.) as previously described (20).

\section{Western blot}

Western blots were performed as described previously $(20,24)$.

\section{Cell proliferation assay}

The cell counting kit-8 (CCK- $\left.8^{\circledR}\right)$ (Dojindo) was used for the cell proliferation assay, according to the manufacturer's instructions. Briefly, at $24 \mathrm{~h}$ after transfection with TagD expression or control vectors, $10^{4}$ cells per well were seeded in 96-well plates and then incubated at $37^{\circ} \mathrm{C}$. After $0,24,48$ and $72 \mathrm{~h}, 10 \mu \mathrm{l}$ of CCK $-8^{\circledR}$ solution was added to each well 
and incubated at $37^{\circ} \mathrm{C}$ for $2 \mathrm{~h}$. The formazan formation was assessed using a plate reader (Nivo) at $450 \mathrm{~nm}$.

\section{Cell migration assay}

Migratory ability of HCT116 cells was examined by wound-healingand transwell migration assays. A scratch wound assay evaluated cell migration and motility. At $24 \mathrm{~h}$ after transfection of TagD expression or control vectors, the transfected cells overgrown in the 33$\mathrm{mm}$ glass-bottom dishes (Iwanami) coated with poly-L-lysin. Then the monolayer cells were scratched by sterile pipette tips uniformly. The cell mobility was monitored for $24 \mathrm{~h}$ using a Keyence BZ-X800 microscope (Keyence, Osaka, Japan) equipped with a time-lapse module. The wound closure areas were measured with Image-J software (1.48q, Rayne Rasband, National Institutes of Health, USA). Cell migratory ability of wound-healing was assessed using the following formula: [(wound area at $0 \mathrm{~h}$ ) - (wound area at indicated $24 \mathrm{~h}$ )] / (wound area at $0 \mathrm{~h}$ ). A higher score indicates a better migratory ability.

In transwell assay, cells were put onto 24 well transwell chambers with a pore size of $8 \mu \mathrm{m}$ (FALCON). The cells had migrated to the reverse face of the membrane. $24 \mathrm{~h}$ after transfection with TagD expression or control vectors, the cells were seeded at $1.5 \times 10^{5}$ cells/well in $200 \mu \mathrm{L}$ medium (supplemented with 0\% FBS) were seeded in the upper chamber. At the same time, $500 \mu \mathrm{L}$ medium (supplemented with $10 \% \mathrm{FBS}$ ) was added in the lower chamber. After incubating for $24 \mathrm{~h}$, the cells on the reverse face were fixed in $4 \%$ paraformaldehyde phosphate buffer solution (Wako) and then stained by $1 \%$ Crystal violet (Sigma-Aldrich) and 2\% ethanol.

\section{Protein expression and purification}

Myc-tagged CDS1, CDS2, PCYT1A, and PCYT1B as well as Flag-tagged PCYT2 and TagD recombinant proteins, were produced by transfecting expression vectors in HCT116 cells. After 48-72 h of transfection, recombinant proteins were purified by anti-c-Myc Antibody Beads (10D11; Wako) or ANTI-FLAG M2 affinity gel (Sigma-Aldrich) following the standard protocol. His6-tagged PCYT $2 \alpha$ or PCYT $2 \beta$ recombinant protein was produced in E. coli BL21-CodonPlus (DE3) (Agilent Technologies), purified by cOmplete His-Tag purification columns (Roche), and concentrated to $1 \mathrm{mg} / \mathrm{mL}$ using Amicon Ultra 10,000 NMWL (Merck). Recombinant $\alpha$-DG 373(T322R)-Fc proteins were prepared from the culture supernatants of the transfected cells as described previously (20).

\section{HPLC analysis of in vitro enzymatic reactions}


CDP-Gro synthetic activity was examined using a mixture of $625 \mu \mathrm{M}$ glycerol-3phosphate, $300 \mu \mathrm{M} \mathrm{CTP}, 1 \mathrm{mM} \mathrm{MgCl} 2,1 \mathrm{mM}$ DTT, and $\sim 12 \mu \mathrm{g} / \mathrm{ml}$ PCYT2 or one of the other candidate enzymes in $4 \mathrm{mM}$ Tris- $\mathrm{HCl}(\mathrm{pH} 7.5)$. The mixture was incubated at $37^{\circ} \mathrm{C}$ overnight. Enzymatic activities of PCYT2 were quantified using a mixture of varying concentrations of ethanolamine phosphate or glycerol-3-phosphate, $300 \mu \mathrm{M} \mathrm{CTP,} 1 \mathrm{mM}$ $\mathrm{MgCl}_{2}, 1 \mathrm{mM}$ DTT, and $72 \mu \mathrm{g} / \mathrm{ml}$ of PCYT $2 \alpha$ or $24 \mu \mathrm{g} / \mathrm{ml}$ of PCYT2 $\beta$ in $4 \mathrm{mM}$ Tris- $\mathrm{HCl}(\mathrm{pH}$ 7.5). The reaction mixture was incubated at $37^{\circ} \mathrm{C}$ for $3 \mathrm{~h}$. After quenching the reaction by boiling at $94^{\circ} \mathrm{C}$ for $5 \mathrm{~min}$, the reaction mixture was two-fold diluted with acetonitrile and subjected to HPLC analysis as described previously (17). CDP-Gro was measured by UV absorbance at $269 \mathrm{~nm}$. The elution positions of CDP-Gro and CDP-ethanolamine were identified using authentic standards (Sigma-Aldrich).

\section{CRISPR/Cas9 genome editing}

The pSpCas9(BB)-2A-Puro (PX459) vector (\#48139) was used for the cloning via BbsI site and the expression of single target gRNAs to generate the KO cells deficient in PCYT2, with target sequences as follows: PCYT2, 5'- CGTTGTCCTTTTCCTAGAGG-3' and 5'GTACAGGTGAGTCTCCACCG-3'. The two constructed pSpCas9(BB)-2A-Puro (PX459) vectors against individual genes were transiently transfected into HCT116 cells in a six-well plate using polyethyleneimine "Max" (Polysciences, Inc.). At $48 \mathrm{~h}$ post-transfection, the cells were treated with puromycin (Thermo Fisher Scientific). Knockout cells were selected with $7.5 \mu \mathrm{g} / \mathrm{ml}$ puromycin for 5 days, then cultured in medium without puromycin for 2-3 weeks. The cells were re-plated as single cells in $6 \mathrm{~cm}$ dishes. Deletions of target genes in the clones were confirmed by PCR, sequencing analysis, and western blotting (Supplemental Figure 1).

\section{Stable cell line generation}

PCYT2-overexpressing cells and PCYT2-KO rescue cells were generated by $600 \mu \mathrm{g} / \mathrm{ml}$ G418 (Thermo Fisher Scientific) selection for 14 days after the transfection with a Flagtagged PCYT2 vector. The expression of PCYT2 in G418-resistant cells was checked by western blot using an anti-PCYT2 antibody (Supplemental Figure 2).

For transposon-mediated gene transfer, cells were transfected with the Tol2 transposase expression vector (pCAGGS-T2TP) and the donor vector (pT2K-CAGGS-rtTA-PCYT2) using PEI-Max. One day after the transfections, cells were selected with $7.5 \mu \mathrm{g} / \mathrm{ml}$ puromycin for 5 days, then cultured in a medium without puromycin for 2 weeks.

\section{LC-MS/MS analysis of nucleotide derivatives}


A HILIC-ESI-MS/MS method was used $(37,38)$ to determine the abundances of CDPglycerol and CDP-ethanolamine in cultured cells. Briefly, cultured cells plated on a 6 -cm dish were collected in ice-cold $70 \%$ ethanol $(1.5 \mathrm{~mL})$, and spiked with an unnatural GDP-glucose $(1 \mathrm{nmol})$. The extracts were centrifuged at $16,000 \mathrm{xg}$ for $15 \mathrm{~min}$ at $4{ }^{\circ} \mathrm{C}$, and the supernatants were lyophilized. As reported previously (37), the samples were dissolved in $2 \mathrm{~mL}$ of $10 \mathrm{mM}$ NH4HCO3 and purified on an Envi-Carb column. The 70\% ethanol extraction precipitate was dissolved in $2 \%$ SDS to measure the protein concentrations, followed by quantifying protein concentration using Pierce BCA Protein Assay Kit (\#23227).

HILIC-ESI-MS/MS was performed on an LCMS-8060 (Shimadzu, Kyoto, Japan) coupled with a Nexera HPLC system (Shimadzu, Kyoto, Japan). Chromatography was performed on a zwitterionic (ZIC) column with phosphocholine phase (ZIC-cHILIC, $2.1 \mathrm{~mm}$ i.d. x $150 \mathrm{~mm}$, $3 \mu \mathrm{m}$; Merck SeQuant, Sweden) (38). CDP-glycerol and CDP-ethanolamine were analyzed in the multiple reaction monitoring mode using specific precursor ion[M-H]- and product ions pairs as follows: CDP-glycerol $m / z 476.2 \rightarrow 322.3$; CDP-ethanolamine, $m / z$ 447.4 $\rightarrow 112.2$. The metabolite levels were normalized as $\mathrm{pmol} / \mathrm{mg}$ proteins.

\section{LC-MS/MS analysis of glycopeptides}

Glycopeptides derived from $\alpha$-DG were identified by LC-MSMS analyses as previously described $(20,24)$. Briefly, the Fc-fused $\alpha$-DG [ $\alpha$-DG 373(T322R)-Fc] were expressed in HCT116 and the PCYT2-KO cells. After purification of the recombinant proteins followed by separation using SDS-PAGE, the gel bands containing $\alpha$-DG373(T322R)-Fc were excised and subjected to reduction, alkylation and then in-gel digestion by trypsin (Promega).

Digested peptides purified using a ZipTip C18 (Merck Millipore) were analyzed by LCMS/MS on an Orbitrap Fusion Tribrid (Thermo Fisher Scientific) coupled to an Easy-nLC 1200 (Thermo Fisher Scientific). The identities of glycopeptides were then manually verified based on positive detection of the expected peptide ${ }^{\text {pyr }}$ QIHATPTPVR $b$ and $y$ ions, and/or the peptide core ion at $\mathrm{m} / \mathrm{z} 551.804(\mathrm{z}=2)$ or $1102.600(\mathrm{z}=1)$ in their respective HCD MS/MS spectra, as described previously $(20,24)$.

\section{Acknowledgments}

We thank Koichi Kawakami (National Institute of Genetics of Japan) for plasmids of pT2KCAGGS-EGFP and pCAGGS-T2TP. We thank Kiyomi Senda (Nagoya City University) for her technical supports. This work was supported in part by Grants-in-Aid for Scientific 
Research (Grant Numbers JP17H06414 to H.Y. and JP24249002 and JP25102008 to K.K.), AMED-Prime under Grant Number 21gm6410010h0001 (to H.Y.), Joint Research of the Exploratory Research Center on Life and Living Systems (ExCELLS) (ExCELLS program No. 20-308 to H.Y. and No. 21-302 to K.H.K.), and an Academia Sinica Investigator Award grant AS-IA-105-L02 to K.H.K.). Mass spectrometry data were acquired at the Academia Sinica Common Mass Spectrometry Facilities for Proteomics and Protein Modification Analysis (supported by grant AS-CFII-108-107). The authors would like to thank Enago (www.enago.jp) for the English language review.

\section{Contributions}

Conceived and designed experiments: H.Y. and K. Kato.

Performed the enzymological experiments: F.U. and H.Y.

Performed the cell-based assay: F.U. and H.Y.

Performed the immunohistochemical experiments: M.N., S.F., F.Y., H.K., and T.S.

Performed the MS experiments: K.N., C.K., and K. Khoo

Wrote a paper: F.U., H.Y., and K. Kato

\section{Conflict of interest}

The authors declare no conflict of interest.

\section{Corresponding author}

Correspondence to H.Y. and K. Kato.

\section{Reference}

1. J. Winkler, A. Abisoye-Ogunniyan, K. J. Metcalf, Z. Werb, Concepts of extracellular matrix remodelling in tumour progression and metastasis. Nat. Commun. 2020111 11, $1-19(2020)$.

2. D. A. Stewart, C. R. Cooper, R. A. Sikes, Changes in extracellular matrix (ECM) and ECM-associated proteins in the metastatic progression of prostate cancer. Reprod. Biol. Endocrinol. 2, 2 (2004).

3. M. Bozzi, F. Sciandra, A. Brancaccio, Role of gelatinases in pathological and physiological processes involving the dystrophin-glycoprotein complex. Matrix Biol., 130-137 (2015). 
4. J. Ervasti, K. Ohlendieck, S. Kahl, M. Gaver, K. Campbell, Deficiency of a glycoprotein component of the dystrophin complex in dystrophic muscle. Nature $\mathbf{3 4 5}$, 315-319 (1990).

5. Y. Hara, et al., Like-acetylglucosaminyltransferase (LARGE)-dependent modification of dystroglycan at Thr-317/319 is required for laminin binding and arenavirus infection. Proc. Natl. Acad. Sci. U. S. A. 108, 17426-17431 (2011).

6. H. Manya, T. Endo, Glycosylation with ribitol-phosphate in mammals: New insights into the O-mannosyl glycan. Biochim. Biophys. acta. Gen. Subj. 1861, 2462-2472 (2017).

7. T. Yoshida-Moriguchi, K. P. Campbell, Matriglycan: a novel polysaccharide that links dystroglycan to the basement membrane. Glycobiology 25, 702-713 (2015).

8. K. Inamori, et al., Dystroglycan Function Requires Xylosyl- and Glucuronyltransferase Activities of LARGE. Science 335, 93 (2012).

9. E. Stevens, et al., Mutations in B3GALNT2 Cause Congenital Muscular Dystrophy and Hypoglycosylation of $\alpha$-dystroglycan. Am. J. Hum. Genet. 92, 354-365 (2013).

10. H. Yagi, et al., AGO61-dependent GlcNAc modification primes the formation of functional glycans on $\alpha$-dystroglycan. Sci. Rep. 3, 3288-3288 (2013).

11. S. Di Costanzo, et al., POMK mutations disrupt muscle development leading to a spectrum of neuromuscular presentations. Hum. Mol. Genet. 23, 5781-5792 (2014).

12. T. Yoshida-Moriguchi, et al., SGK196 Is a Glycosylation-Specific $O$-Mannose Kinase Required for Dystroglycan Function. Science 341, 896-899 (2013).

13. T. Willer, et al., The glucuronyltransferase B4GAT1 is required for initiation of LARGE-mediated $\alpha$-dystroglycan functional glycosylation. Elife 3, e03941 (2014).

14. J. L. Praissman, et al., B4GAT1 is the priming enzyme for the LARGE-dependent functional glycosylation of $\alpha$-dystroglycan. Elife 3, 3943 (2014).

15. M. Kanagawa, et al., Identification of a Post-translational Modification with RibitolPhosphate and Its Defect in Muscular Dystrophy. Cell Rep. 14, 2209-2223 (2016).

16. H. Manya, et al., The Muscular Dystrophy Gene TMEM5 Encodes a Ribitol $\beta 1,4-$ Xylosyltransferase Required for the Functional Glycosylation of Dystroglycan. J. Biol. Chem. 291, 24618-24627 (2016). 
17. M. Riemersma, et al., Human ISPD Is a Cytidyltransferase Required for Dystroglycan O-Mannosylation. Chem. Biol. 22, 1643-1652 (2015).

18. T. Willer, et al., ISPD loss-of-function mutations disrupt dystroglycan Omannosylation and cause Walker-Warburg syndrome. Nat. Genet. 44, 575-580 (2012).

19. I. Gerin, et al., ISPD produces CDP-ribitol used by FKTN and FKRP to transfer ribitol phosphate onto $\alpha$-dystroglycan. Nat. Commun. 7, 11534-11534 (2016).

20. H. Yagi, et al., Direct Mapping of Additional Modifications on Phosphorylated $O$ glycans of $\alpha$-Dystroglycan by Mass Spectrometry Analysis in Conjunction with Knocking Out of Causative Genes for Dystroglycanopathy. Mol. Cell. Proteomics 15, 3424-3434 (2016).

21. R. Imae, et al., CDP-glycerol inhibits the synthesis of the functional $O$-mannosyl glycan of $\alpha$-dystroglycan. J. Biol. Chem. 293, 12186-12198 (2018).

22. Z. Qian, et al., Genomic characterization of ribitol teichoic acid synthesis in Staphylococcus aureus: genes, genomic organization and gene duplication. BMC Genomics 7, 74 (2006).

23. A. Formstone, R. Carballido-López, P. Noirot, J. Errington, D. J. Scheffers, Localization and Interactions of Teichoic Acid Synthetic Enzymes in Bacillus subtilis. J. Bacteriol. 190, 1812-1821 (2007).

24. F. Yamasaki, et al., Establishment of a novel monoclonal antibody against truncated glycoforms of $\alpha$-dystroglycan lacking matriglycans. Biochem. Biophys. Res. Commun. 579, 8-14 (2021).

25. J. Muschler, et al., A Role for Dystroglycan in Epithelial Polarization: Loss of Function in Breast Tumor Cells. CANCER Res. 62, 7102-7109 (2002).

26. M. Imperiali, et al., O Mannosylation of $\alpha$-Dystroglycan Is Essential for Lymphocytic Choriomeningitis Virus Receptor Function. J. Virol. 79, 14297-14308 (2005).

27. O. B. Bleijerveld, W. Klein, A. B. Vaandrager, J. B. Helms, M. Houweling, Control of the CDPethanolamine pathway in mammalian cells: effect of CTP:phosphoethanolamine cytidylyltransferase overexpression and the amount of intracellular diacylglycerol. Biochem. J. 379, 711 (2004).

28. Z. Pavlovic, M. Bakovic, Regulation of Phosphatidylethanolamine Homeostasis-The Critical Role of CTP: Phosphoethanolamine Cytidylyltransferase (Pcyt2). Int. J. Mol. Sci. 14, 2529-2550 (2013). 
29. J. Y. Zhou, D. M. Oswald, K. D. Oliva, L. S. C. Kreisman, B. A. Cobb, The Glycoscience of Immunity. Trends Immunol. 39, 523-535 (2018).

30. R. Kleene, M. Schachner, Glycans and neural cell interactions. Nat. Rev. Neurosci. 5, 195-208 (2004).

31. S. S. Pinho, C. A. Reis, Glycosylation in cancer: mechanisms and clinical implications. Nat. Rev. Cancer 2015159 15, 540-555 (2015).

32. K. T. Schjoldager, Y. Narimatsu, H. J. Joshi, H. Clausen, Global view of human protein glycosylation pathways and functions. Nat. Rev. Mol. Cell Biol. 20202112 21, 729-749 (2020).

33. X. Bao, et al., Tumor suppressor function of laminin-binding $\alpha$-dystroglycan requires a distinct $\beta 3-N$-acetylglucosaminyltransferase. Proc. Natl. Acad. Sci. 106, 12109-12114 (2009).

34. M. D. Fullerton, F. Hakimuddin, M. Bakovic, Developmental and Metabolic Effects of Disruption of the Mouse CTP:Phosphoethanolamine Cytidylyltransferass Gene (Pcyt2). Mol. Cell. Biol. 27, 3327-3336 (2007).

35. L.-Y. Liou, et al., Functional Glycosylation of Dystroglycan Is Crucial for Thymocyte Development in the Mouse. PLoS One 5 (2010).

36. R. Imae, H. Manya, H. Tsumoto, Y. Miura, T. Endo, PCYT2 synthesizes CDPglycerol in mammals and reduced PCYT2 enhances the expression of functionally glycosylated $\alpha$-dystroglycan. J. Biochem. 170, 183-194 (2021).

37. Y. Harada, K. Nakajima, S. Li, T. Suzuki, N. Taniguchi, Protocol for analyzing the biosynthesis and degradation of N-glycan precursors in mammalian cells. STAR Protoc. 2, 100316-100316 (2021).

38. J. Ito, et al., Analysis of plant nucleotide sugars by hydrophilic interaction liquid chromatography and tandem mass spectrometry. Anal. Biochem. 448, 14-22 (2014). 


\section{Figure legends}

\section{Figure 1. Immunohistochemical analysis for GroP modification in human colorectal} cancer tissues. (A) Representative images of staining with DG2. The left and right panels represent negative and positive DG2 staining, respectively, in human colorectal cancer tissues (x100). (B) GroP expression according to disease stage. Each bar represents the DG2 positive rate in each stage of colorectal cancer. *, $P<0.05 ; * *, P<0.01$

Figure 2. Effects of enhancement of the GroP modification on cancer cell behaviors. (A) TagD overexpression reduced the expression of laminin-binding glycans. Cell lysates containing equal amounts of total proteins prepared from HCT116 cells transfected with TagDexpressing vector or control vector were subjected to immunoblot analysis using IIH6 and anti-Flag antibody. (B) Proliferation assay of HCT116 cells transfected with TagDexpressing vector or control vector at $0,24,48$, and $72 \mathrm{~h}$. Error bars represent the standard error of the mean $(\mathrm{SEM})(\mathrm{n}=3)$. (C) Transwell assay (scale bar $=600 \mu \mathrm{m})$ and (D) Woundhealing assay (scale bar $=200 \mu \mathrm{m}$ ) for HCT116 cells at $24 \mathrm{~h}$ after transfection with TagDexpressing or control vector. (E) Wound-healing index of TagD-overexpressing cells and control cells. Error bars represent the SEM $(n=3)$. Significant differences $(*)$ were calculated compared with WT index using two-tailed unpaired Student's t test $(P<0.05)$.

Figure 3. Characterization of enzymatic activities of PCYT2. (A) HPLC profiles of the reaction mixtures in the presence and absence of the candidate enzymes listed in Table 1 and positive (TagD) and negative controls. The peak corresponding to CDP-Gro is indicated with arrow. (B) The enzymatic activities of PCYT2 $\alpha$ and PCYT2 $\beta$ with various glycerol-3phosphate or ethanolamine phosphate and CTP concentrations are plotted. CDP-Gro/CDP- 
Etn synthase activities of PCYT2 $\alpha(\boldsymbol{\nabla} / \mathbf{O})$ and PCYT2 $\beta(\bullet / \mathbf{\Delta})$ were represented, respectively. The data were fitted to the Michaelis-Menten equation by non-linear regression (GraphPad Prism 9; https://www.graphpad.com/scientific-software/prism/). The apparent Michaelis constant $\left(K_{\mathrm{m}}\right)$ and the maximal velocity $\left(V_{\max }\right)$ were calculated.

Figure 4. LC-ESI-MS/MS analysis of CDP-Gro and CDP-Etn from HCT116 cells (A) Representative extraction ion chromatograms of CDP-Gro (Upper panel) and CDP-Etn (Lower panel) derived from HCT116. (B) HCT116 wild-type cells, TagD-overexpressing

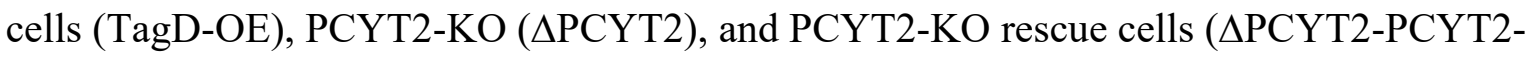
$\mathrm{OE}$ ) were analyzed, and the data were normalized to units of pmol per milligram of protein. Error bars represent the standard error of the mean $(\mathrm{SEM})(\mathrm{n}=3)$.

Figure 5. Glycan modification on $\alpha$-DG373(T322R)-Fc expressed in HCT116 wild-type and PCYT2-KO cells. Overlays of the extracted ion chromatograms of select ${ }^{313}$ pyrQIHATPTPVR ${ }^{322}$ glycoforms carrying none and phosphorylated core M3 substituents of WT (Upper panel) and $\triangle$ PCYT2 (Lower panel) derived from HCT116.

Figure 6. The expression of laminin-binding glycans was reduced by PCYT2-

overexpression. HCT116 clone stably transfected with the doxycycline (Dox)-inducible PCYT2 construct; - Dox, uninduced cells; + Dox, induced cells. Cell lysates containing equal amount of total proteins prepared from HCT116 cells with $(+)$ or without $(-)$ Dox were subjected to immunoblot analysis using anti-Flag antibody and anti- $\beta$-actin, or to WGA enrichment followed by immunoblot analysis using IIH6. 


\section{Figure 7. Correlation between GroP modification and PCYT2 expression levels in}

colorectal cancer tissues. (A) Representative images of PCYT2 staining. The left and right panels represent negative PCYT2 and positive PCYT2 staining, respectively, in human colorectal cancer tissues (x100). (B) Mapping of correlation between GroP modification and PCYT2 expression levels based on the staining scores. Staining scores of both GroP and PCYT2 were calculated at stage IV colorectal cancer tissues, and data were analyzed using the Spearman rank correlation.

Table 1: Sequence identities of homologs genes with the TagD gene calculated utilizing the BLAST 2.0 algorithm

Gene Protein Identity Query cover

\begin{tabular}{cccc}
\hline CDS1 & Phosphatidate cytidylyltransferase 1 & $57 \%$ & $10 \%$ \\
CDS2 & Phosphatidate cytidylyltransferase 2 & $67 \%$ & $8 \%$ \\
PCYT1A & Choline-phosphate cytidylyltransferase A & $35 \%$ & $93 \%$ \\
PCYT1B & Choline-phosphate cytidylyltransferase B & $36 \%$ & $93 \%$ \\
PCYT2 & Ethanolamine-phosphate cytidylyltransferase & $30 \%$ & $89 \%$ \\
\hline
\end{tabular}


Table 2: The enzymatic parameters of CDP-Gro or CDP-Etn synthase activities of

PCYT2 isoforms using glycerol-3-phosphate or ethanolamine phosphate as substrates,

respectively.

CDP-Etn

PCYT2 $\alpha$

$V_{\max }(\mathrm{mM} / \mathrm{h})$

$K_{\mathrm{m}}(\mathrm{mM})$

Catalytic efficiency

$\left(k_{\text {cat }} / K_{\mathrm{m}}\right)\left(\mathrm{mM}^{-1} \mathrm{~h}^{-1}\right)$
33.7

181.2

0.3530

0.3974

37.3

171.2
CDP-Gro

PCYT2 $\alpha$

22.7

PCYT2 $\beta$
1614

2899

0.0089

0.0060 
A

Negative

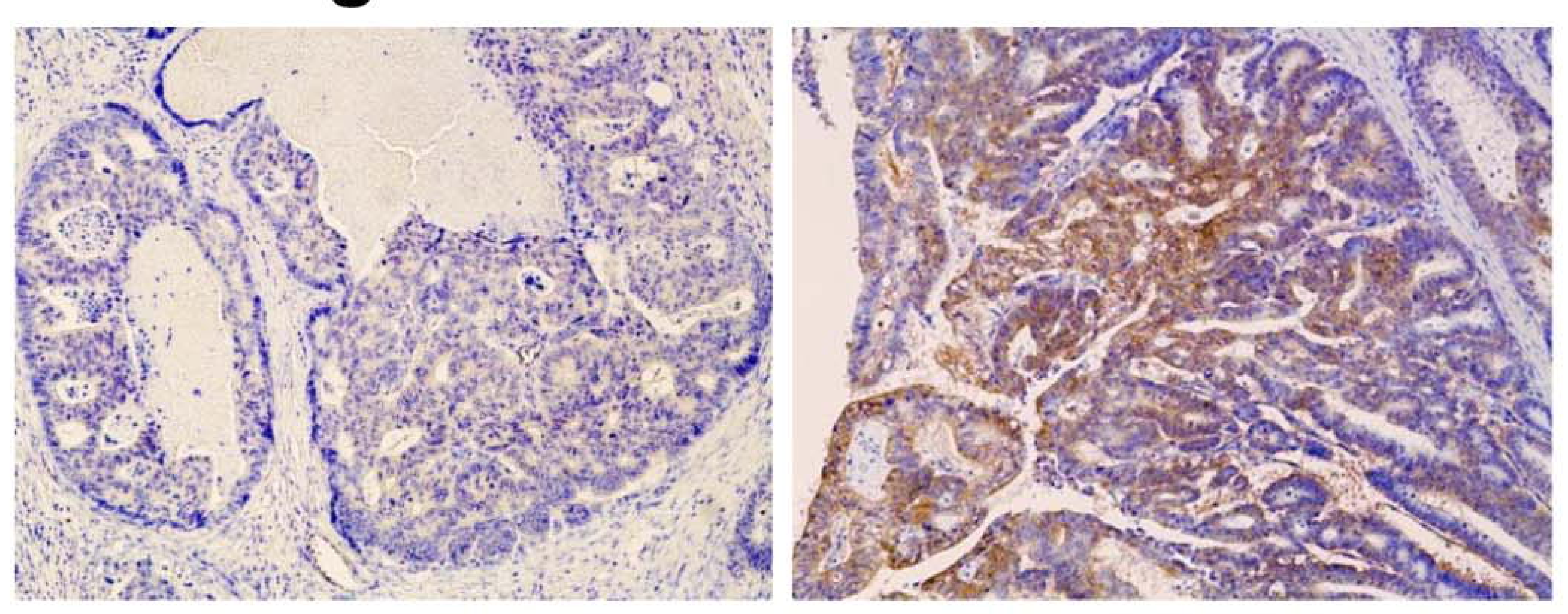

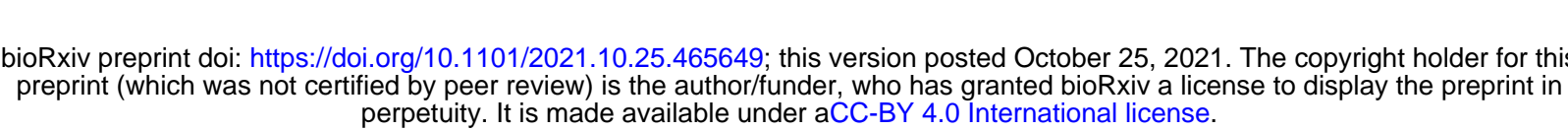

B

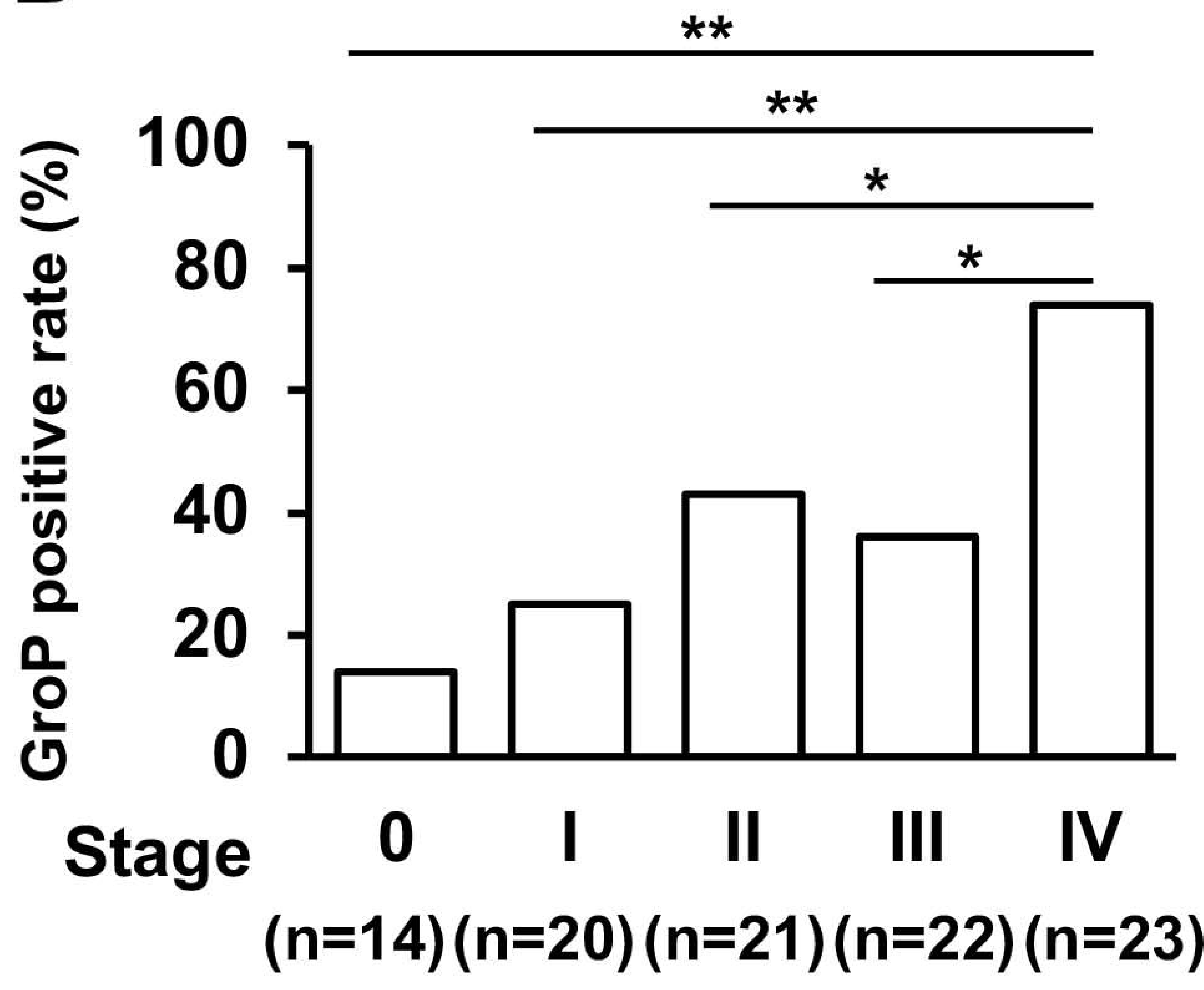




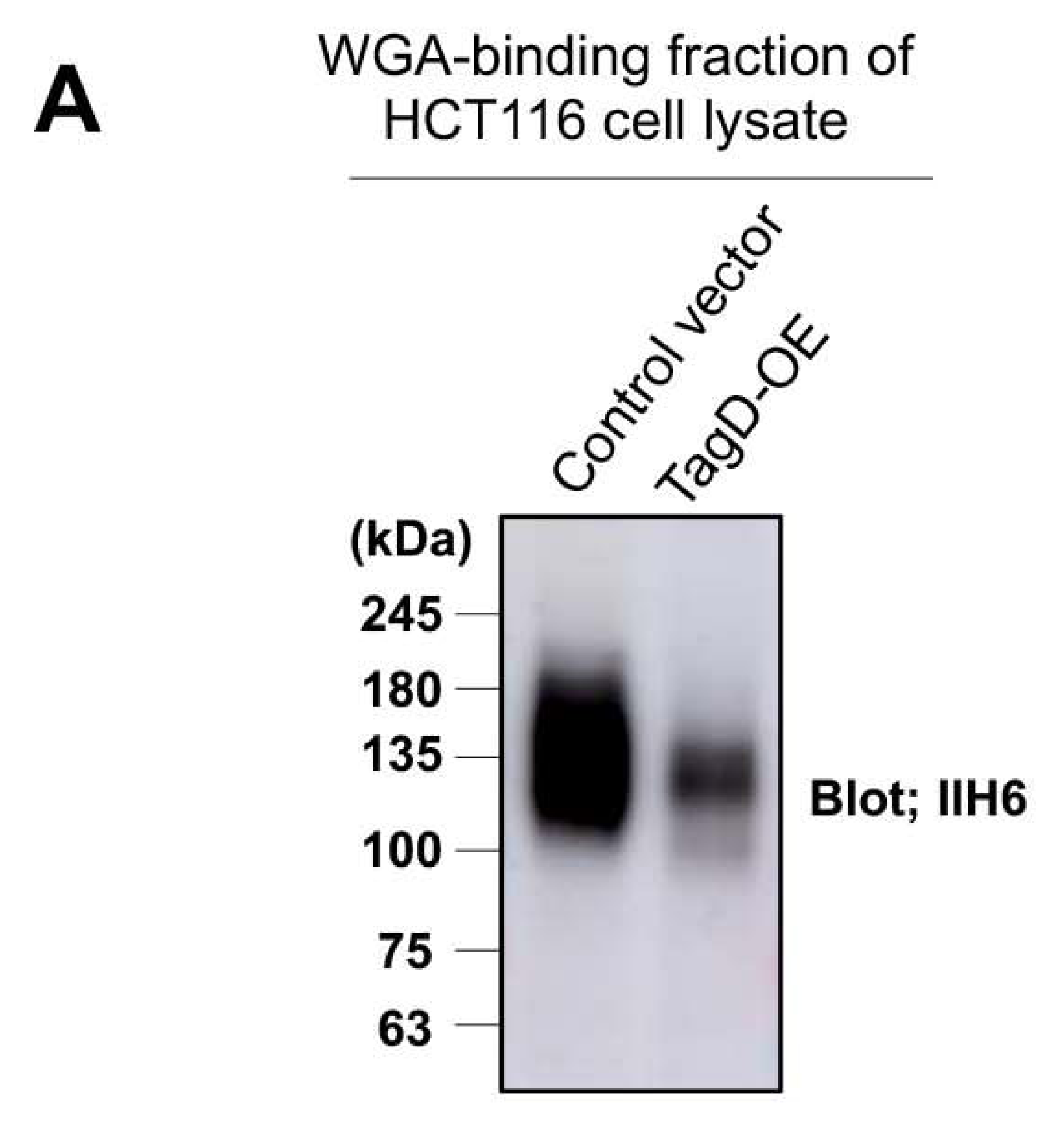

HCT116 cell lysate
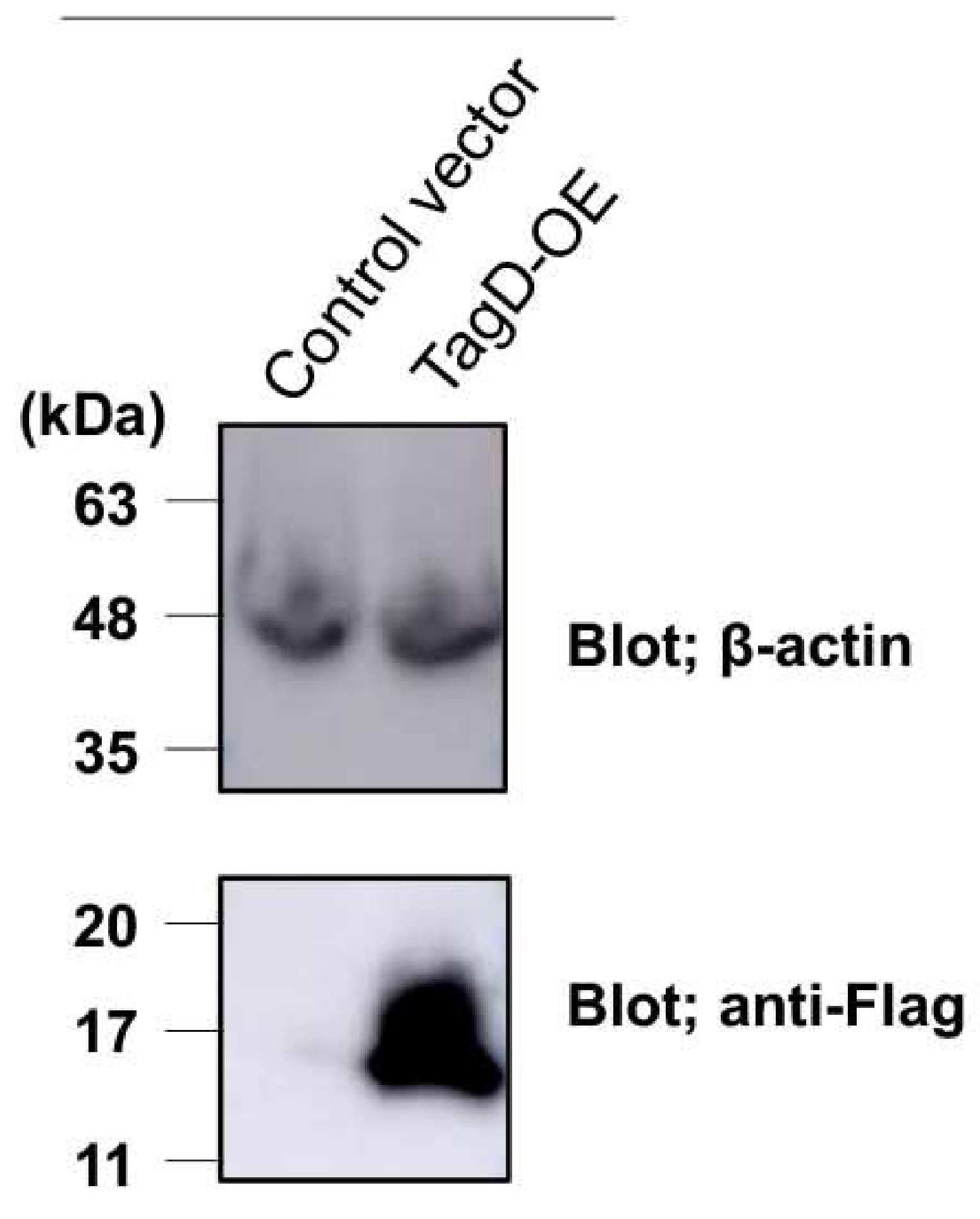

C

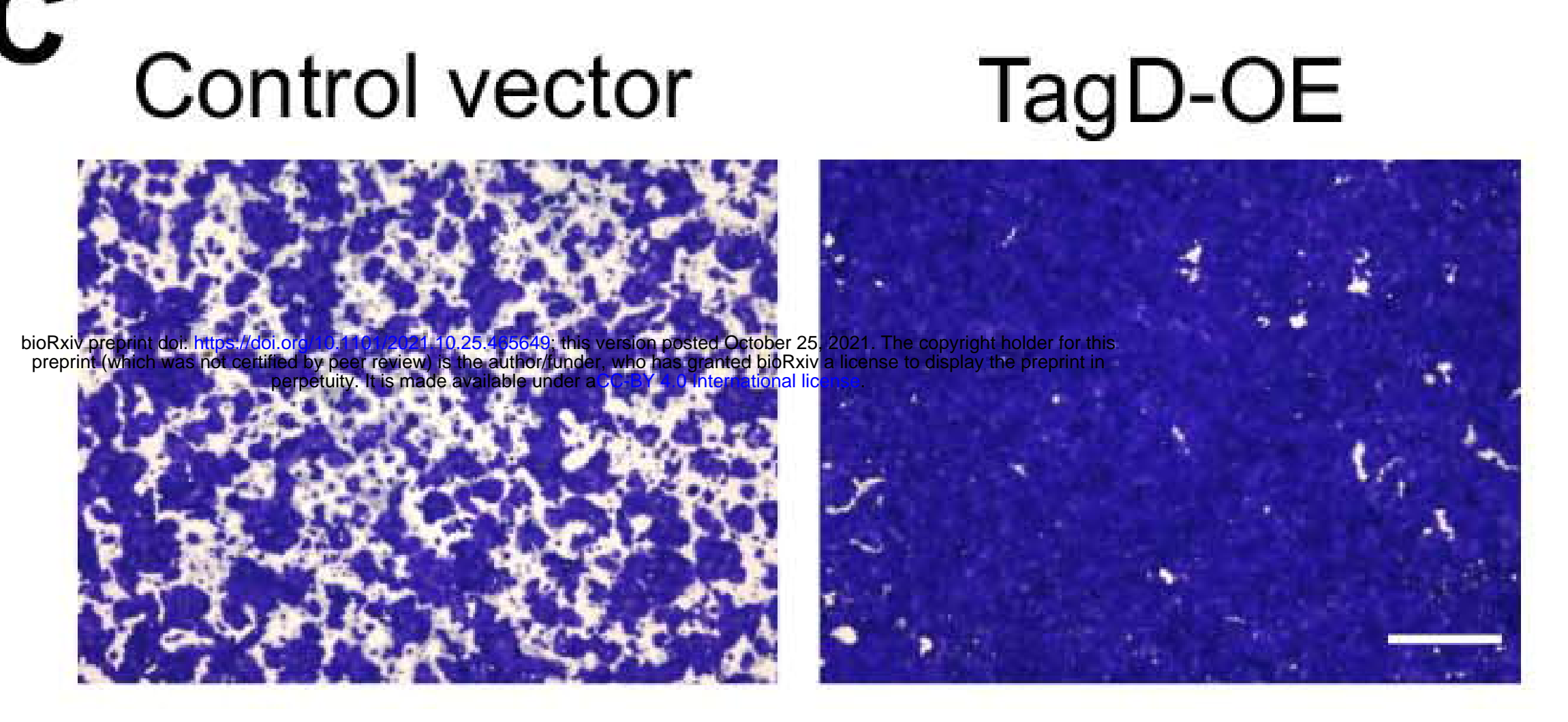

B
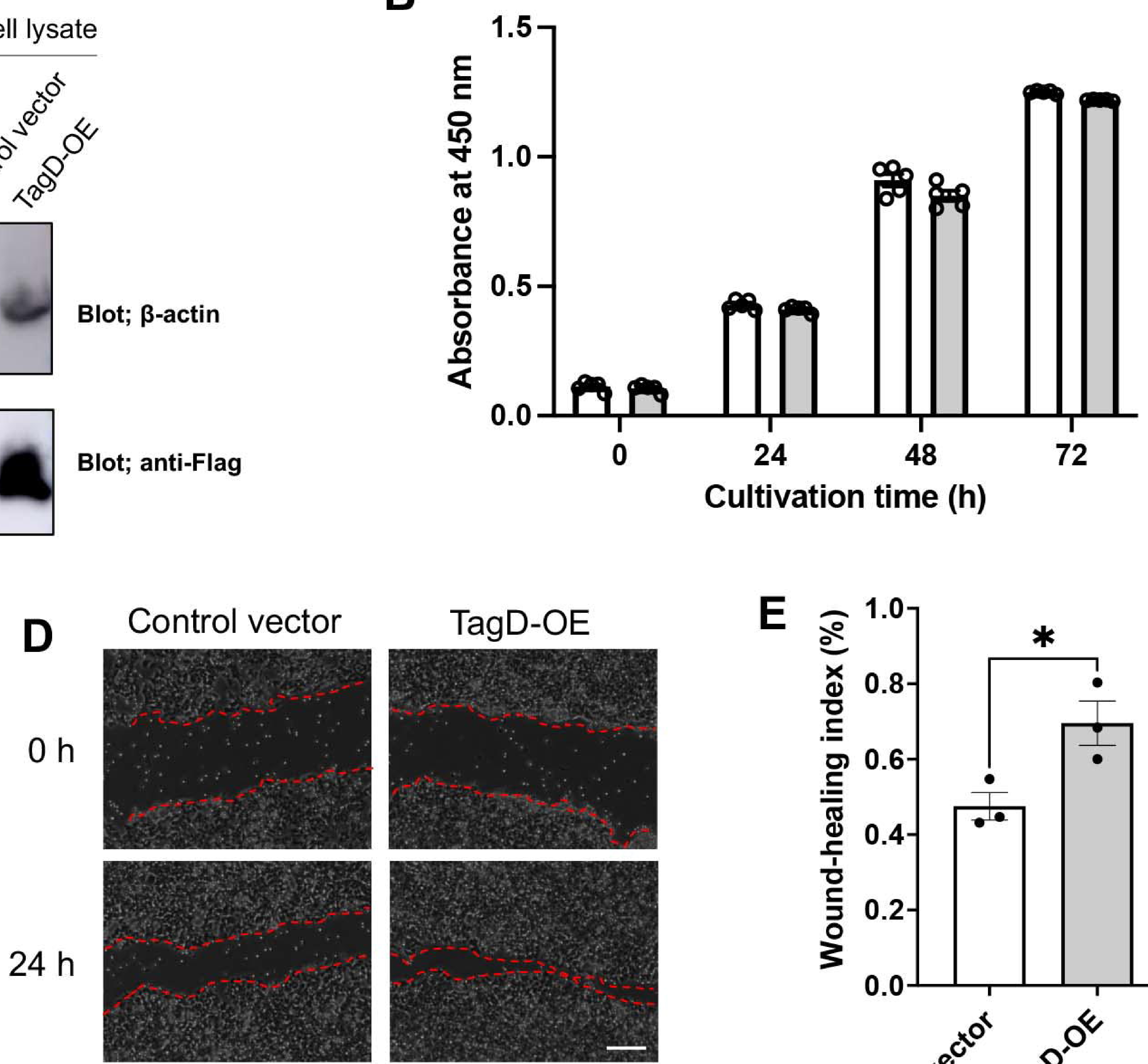

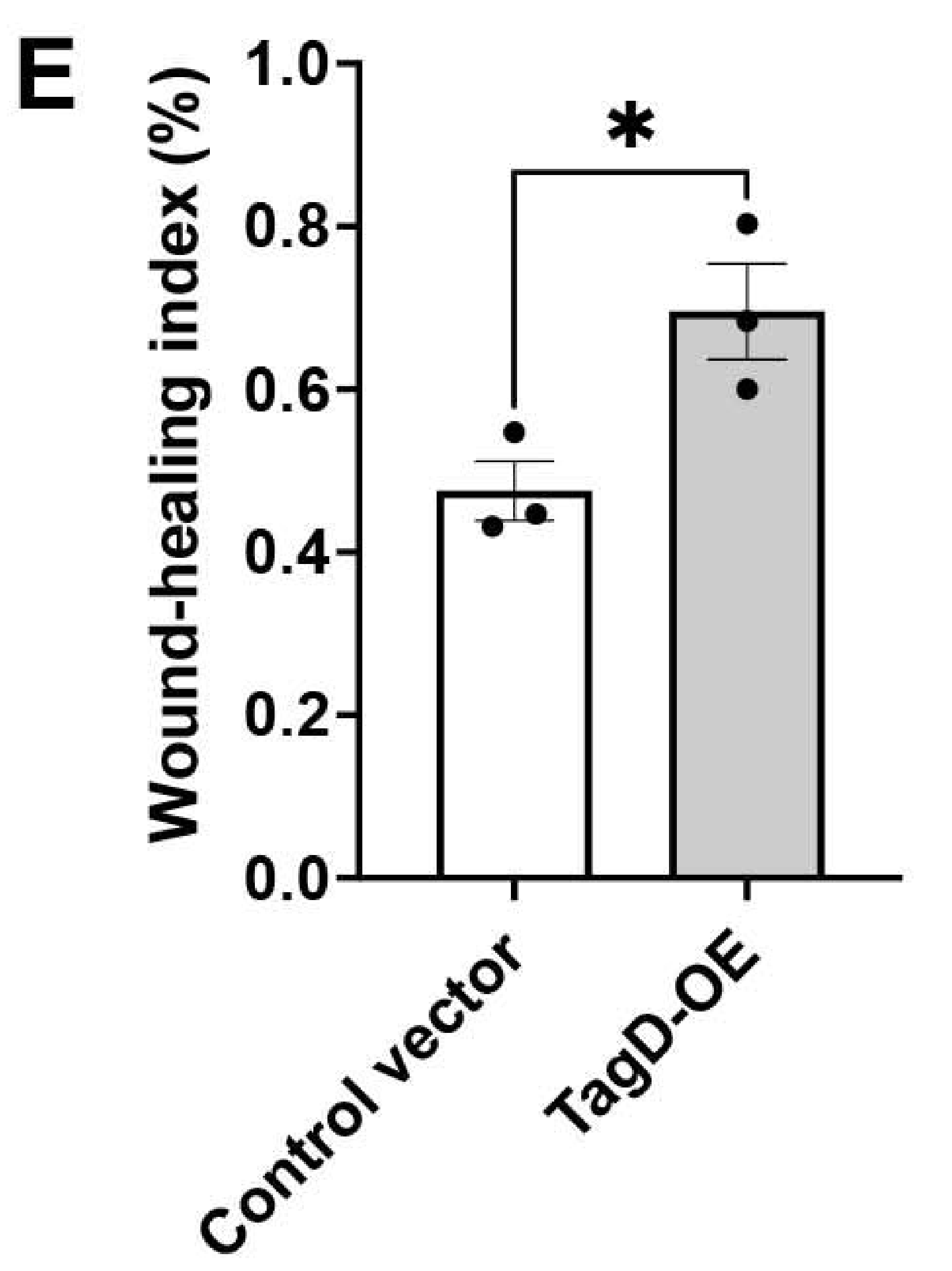


A

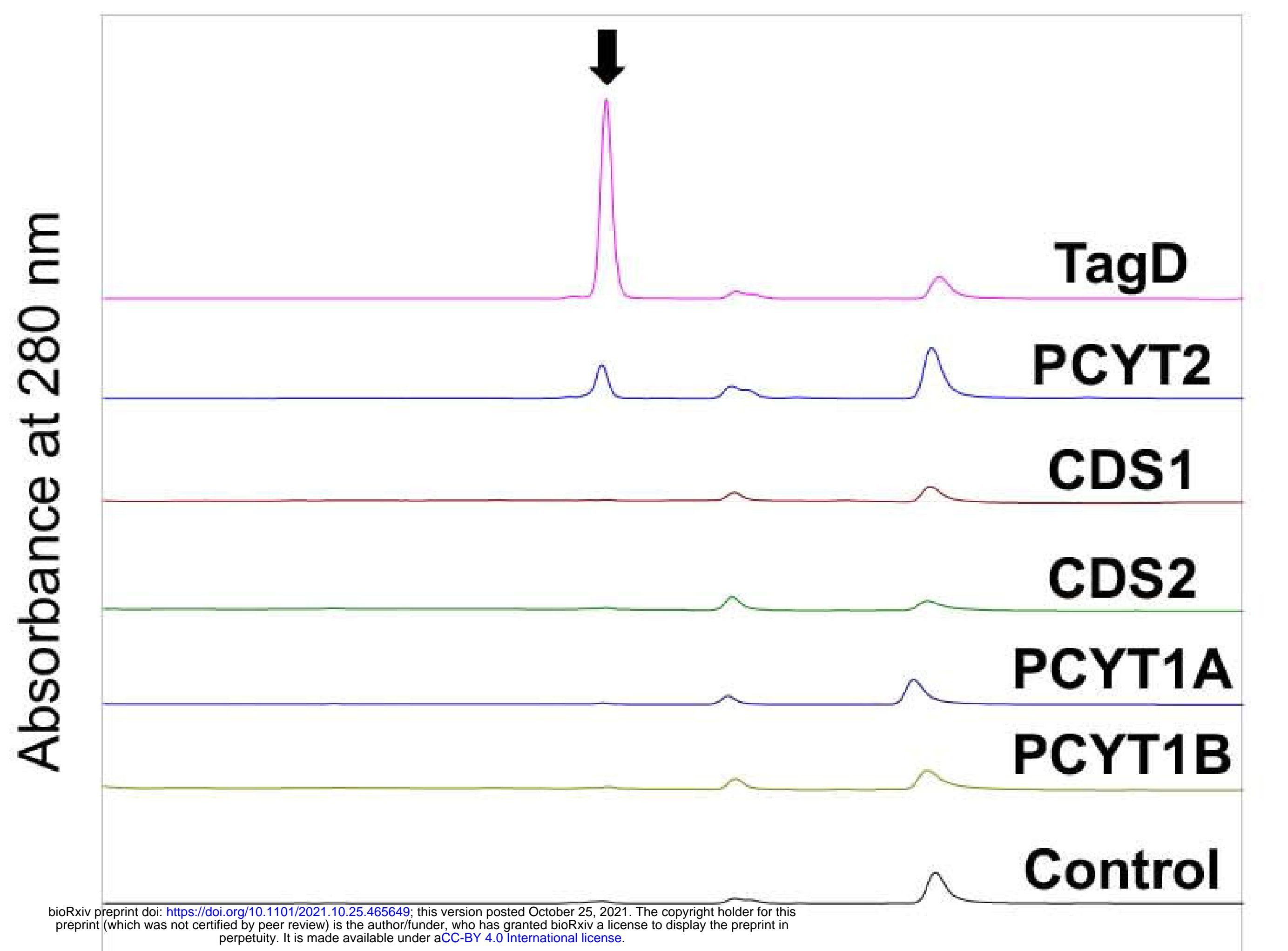

$\begin{array}{llllllll}8 & 9 & 10 & 11 & 12 & 13 & 14 & 15\end{array}$

Elution time $(\mathrm{min})$
B
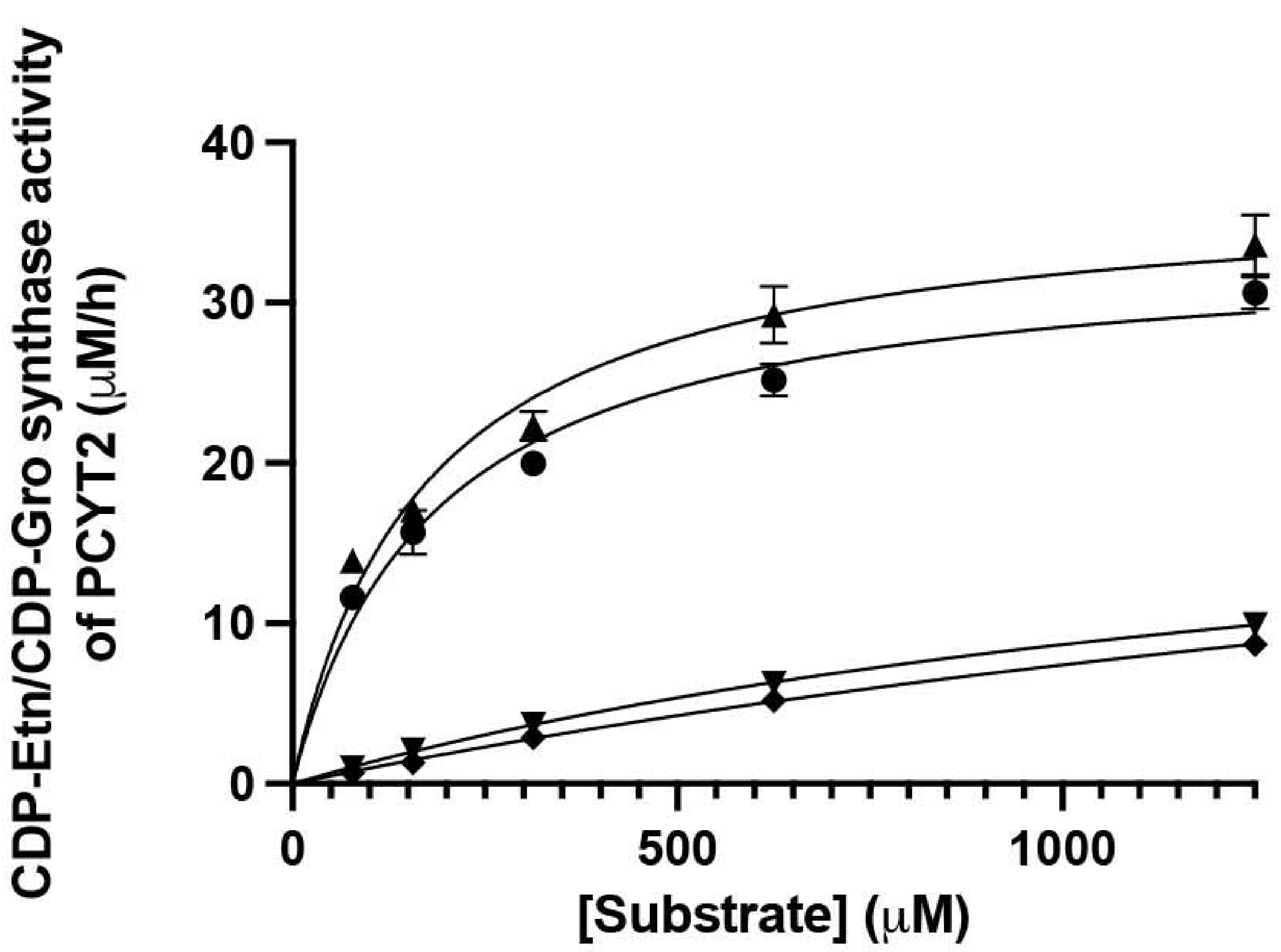

$\rightarrow$ PCYT2a/CDP-Etn

$\star$ PCYT2 $\beta / C D P-E t n$

$\rightarrow \mathrm{PCYT} 2 \alpha / C D P-G r o$

$\rightarrow$ PCYT2 $\beta / C D P-G r o$ 
A
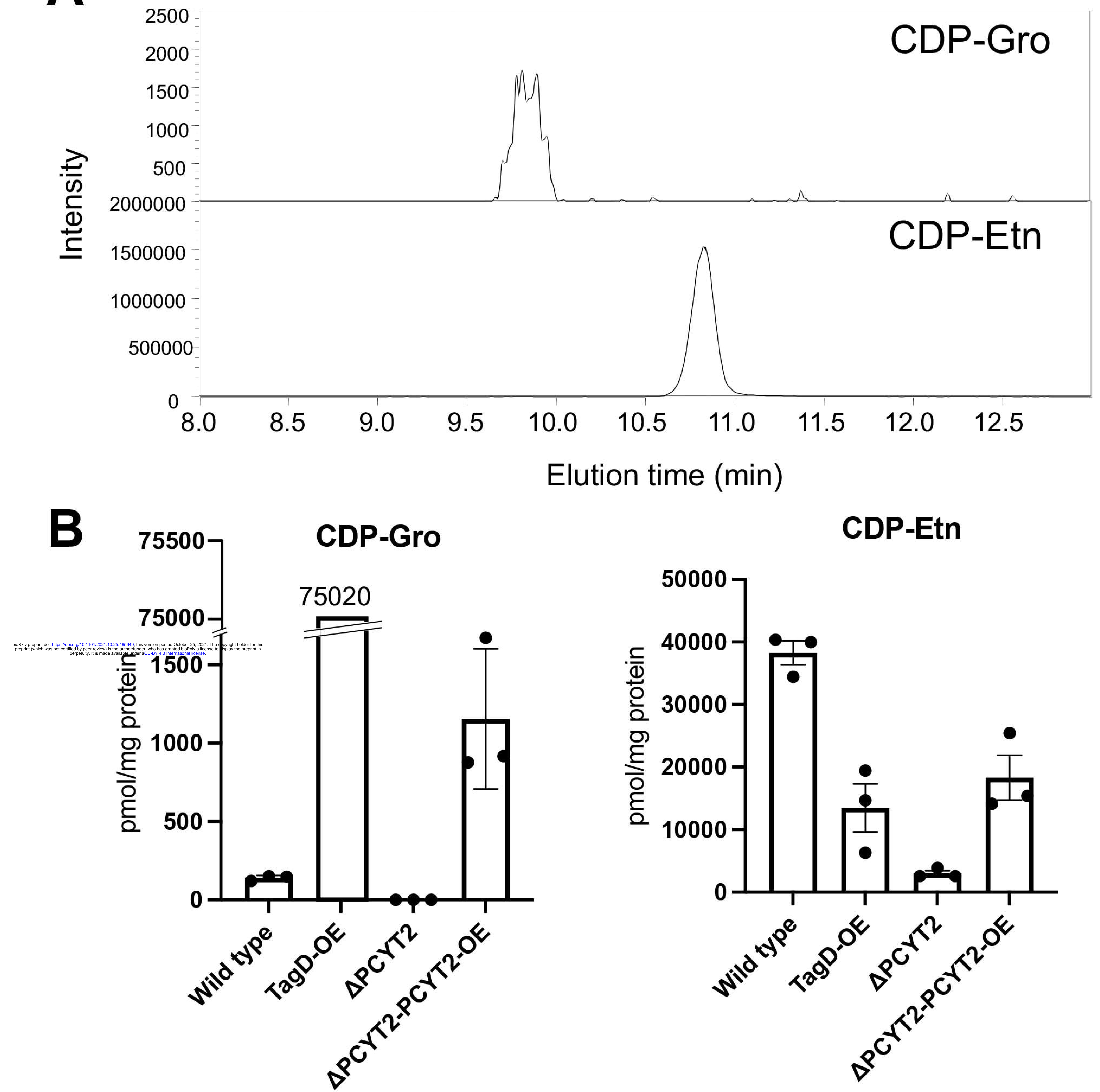

CDP-Etn

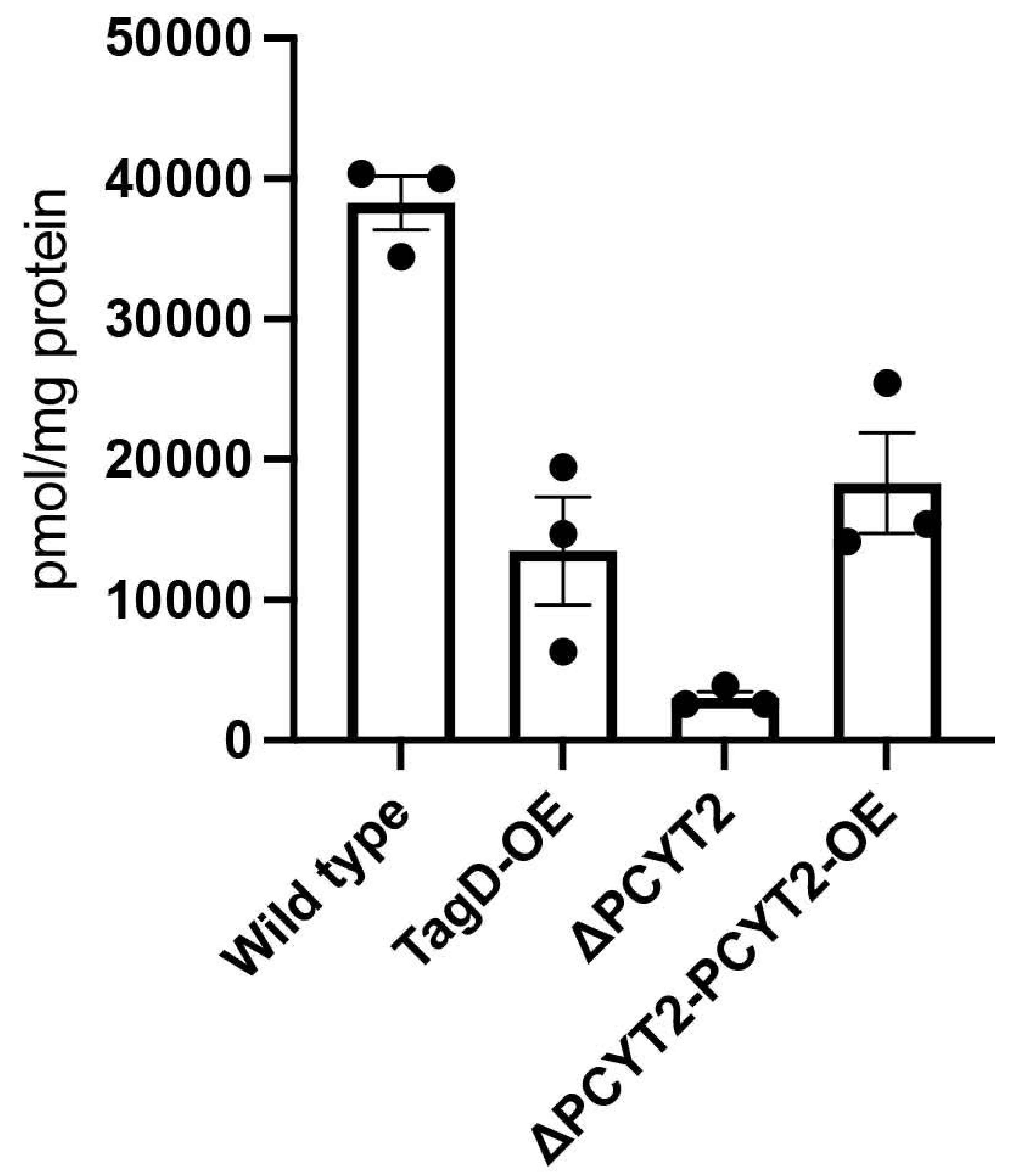




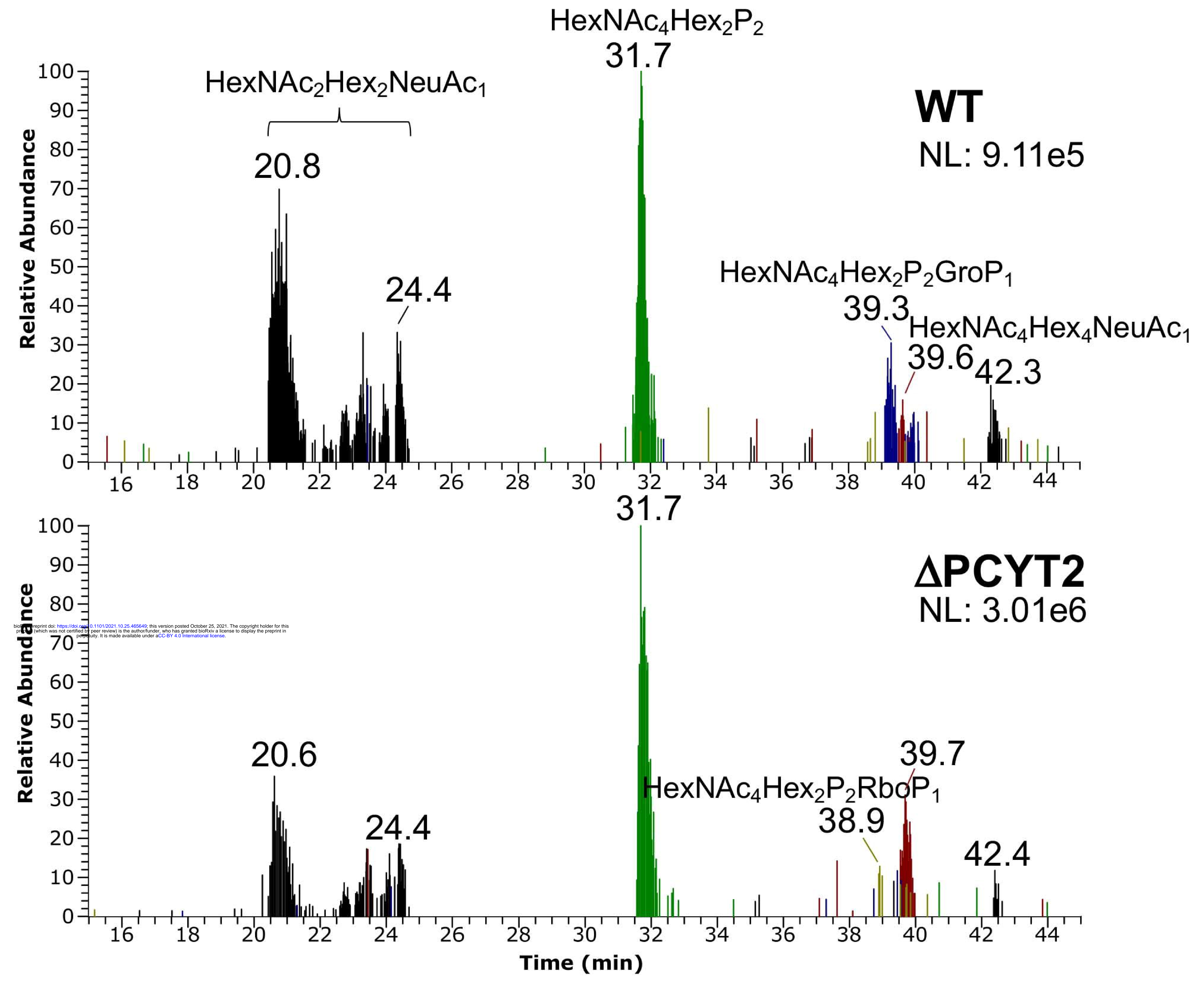


WGA-binding fraction of HCT116 cell lysate

HCT116 cell lysate
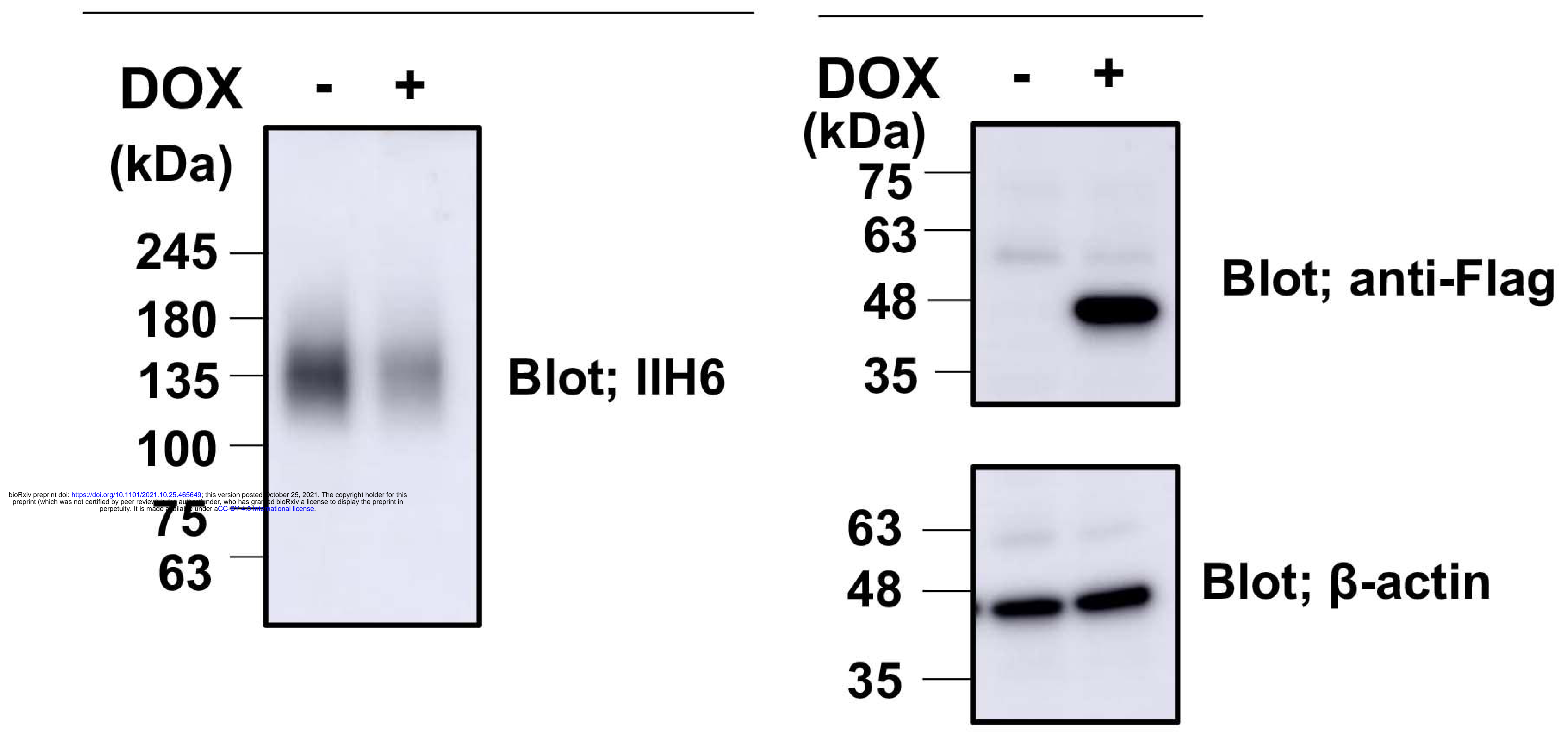
B

Negative

Positive

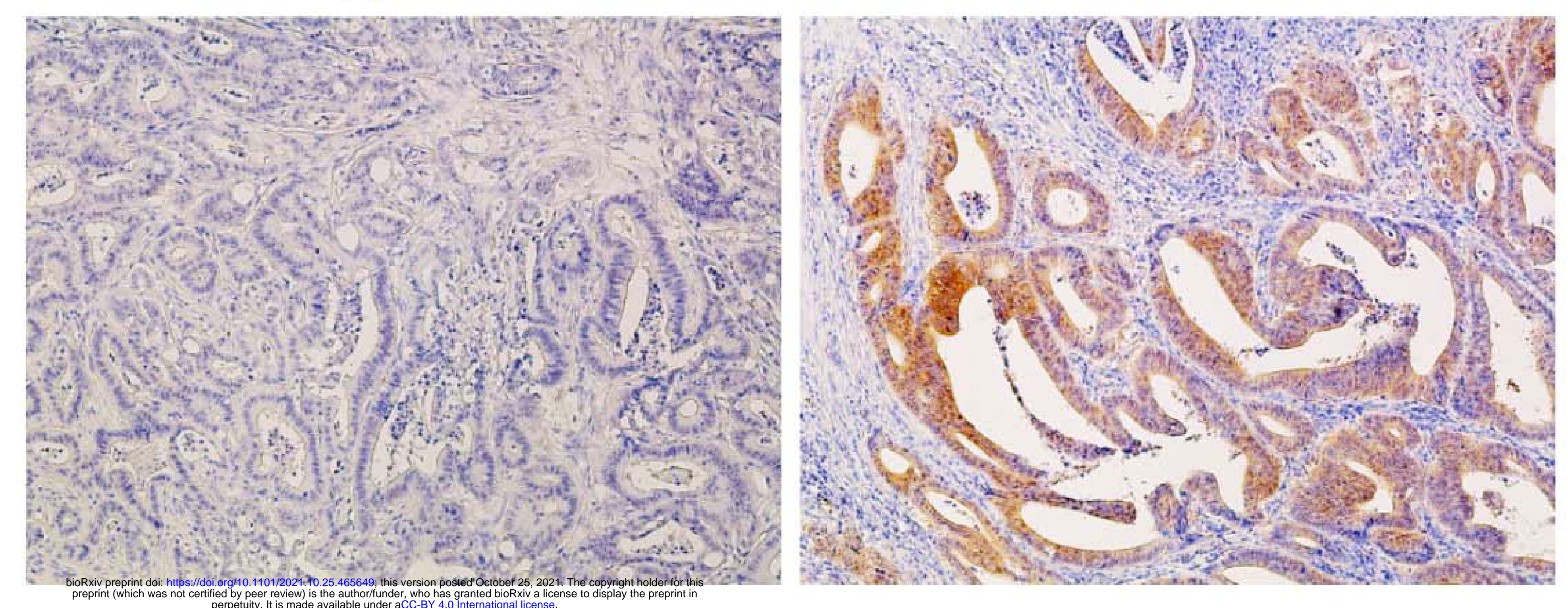

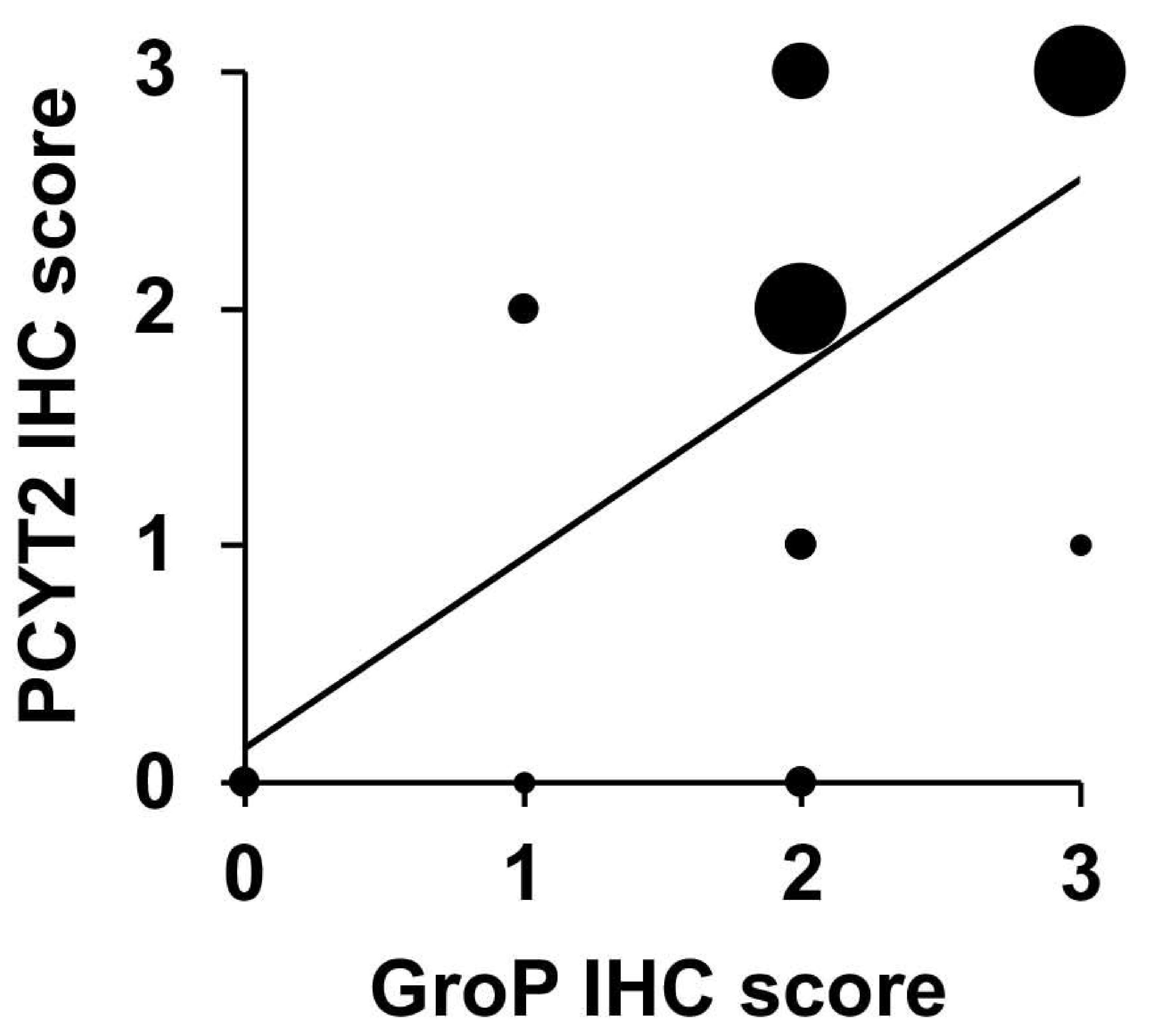

